\title{
Weak Superconducting Pairing and a Single Isotropic Energy Gap in Stoichiometric LiFeAs
}

\author{
D. S. Inosov, ${ }^{1, *}$ J. S. White, ${ }^{2}$ D. V. Evtushinsky, ${ }^{3}$ I. V. Morozov, ${ }^{4,3}$ A. Cameron, ${ }^{5}$ U. Stockert, ${ }^{3}$ V. B. Zabolotnyy, ${ }^{3}$ \\ T. K. Kim, ${ }^{3}$ A. A. Kordyuk, ${ }^{3,6}$ S. V. Borisenko, ${ }^{3}$ E. M. Forgan, ${ }^{5}$ R. Klingeler, ${ }^{3}$ J. T. Park, ${ }^{1}$ S. Wurmehl, ${ }^{3}$ A. N. Vasiliev, ${ }^{4}$ \\ G. Behr, ${ }^{3}$ C. D. Dewhurst, ${ }^{7}$ and V. Hinkov ${ }^{1}$ \\ ${ }^{1}$ Max Planck Institute for Solid State Research, Heisenbergstraße 1, D-70569 Stuttgart, Germany \\ ${ }^{2}$ Laboratory for Neutron Scattering, Paul Scherrer Institut, 5232 Villigen PSI, Switzerland \\ ${ }^{3}$ Leibnitz, Institute for Solid State Research, IFW Dresden, D-01171 Dresden, Germany \\ ${ }^{4}$ Moscow State University, Moscow 119991, Russia \\ ${ }^{5}$ School of Physics and Astronomy, University of Birmingham, Edgbaston, Birmingham, B15 2TT, United Kingdom \\ ${ }^{6}$ Institute for Metal Physics of the National Academy of Sciences of Ukraine, 03142 Kyiv, Ukraine \\ ${ }^{7}$ Institut Laue-Langevin, 6 Rue Jules Horowitz, F-38042 Grenoble, France
}

(Received 20 February 2010; published 6 May 2010)

\begin{abstract}
We report superconducting (SC) properties of stoichiometric LiFeAs $\left(T_{c}=17 \mathrm{~K}\right)$ studied by smallangle neutron scattering (SANS) and angle-resolved photoemission (ARPES). Although the vortex lattice exhibits no long-range order, well-defined SANS rocking curves indicate better ordering than in chemically doped 122 compounds. The London penetration depth $\lambda_{a b}(0)=210 \pm 20 \mathrm{~nm}$, determined from the magnetic field dependence of the form factor, is compared to that calculated from the ARPES band structure with no adjustable parameters. The temperature dependence of $\lambda_{a b}$ is best described by a single isotropic SC gap $\Delta_{0}=3.0 \pm 0.2 \mathrm{meV}$, which agrees with the ARPES value of $\Delta_{0}^{\mathrm{ARPES}}=3.1 \pm$ $0.3 \mathrm{meV}$ and corresponds to the ratio $2 \Delta / k_{B} T_{c}=4.1 \pm 0.3$, approaching the weak-coupling limit predicted by the BCS theory. This classifies LiFeAs as a weakly coupled single-gap superconductor.
\end{abstract}

In many of the recently discovered Fe-based superconductors (SC) [1], a transition to the SC state is induced by chemical doping of a parent compound that at ambient conditions does not exhibit SC in its stoichiometric composition even at the lowest temperatures. Among the few known exceptions, the present record holder for the SC transition temperature $T_{c}$ is the stoichiometric LiFeAs $\left(T_{c} \lesssim 18 \mathrm{~K}\right)$ [2]. Its electronic structure is quasi-twodimensional (2D) [3] and supports superconductivity in the absence of any notable Fermi surface (FS) nesting or static magnetism [4]. However, the presence of normalstate antiferromagnetic fluctuations has been suggested by ${ }^{75}$ As NMR measurements [5]. Together with the weakness of the electron-phonon coupling predicted by the density functional theory [6], this suggests that the SC pairing in this structurally simple compound possibly has the same magnetic origin as in higher- $T_{c}$ iron pnictides [7]. On the other hand, arguments advocating the phonon mechanism have also been raised recently [8]. Therefore, to pinpoint the SC mechanism with certainty, details of the SC pairing symmetry and the coupling strength are required.

In a number of recent studies [9-12], it was shown that doped iron arsenide superconductors are characterized by strong pinning of magnetic-flux lines that precludes the formation of an ordered Abrikosov lattice. The role of the pinning centers can be played by magnetic or structural domains in the underdoped samples [11], by the dopant atoms themselves, such as $\mathrm{Co}$ or $\mathrm{Ni}$, at higher doping levels [12], or by the electronic inhomogeneities that result from phase separation in some hole-doped 122 systems [13].
This served as our motivation to study the magnetic field penetration in a single crystal of stoichiometric LiFeAs, which possesses a nonmagnetic ground state with tetragonal crystal symmetry, thus excluding all of the abovementioned strong pinning mechanisms from consideration. In the following, we will compare these results with angleresolved photoemission (ARPES) measurements of the electronic structure to establish the microscopic origin of the measured quantities.

For our small-angle neutron scattering (SANS) experiments, we used a large single crystal with a volume of $\sim 10 \times 10 \times 0.4 \mathrm{~mm}^{3}$. It was grown by the self-flux method and characterized as described in Ref. [4]. The value of $T_{c}$, measured on a smaller piece of the same
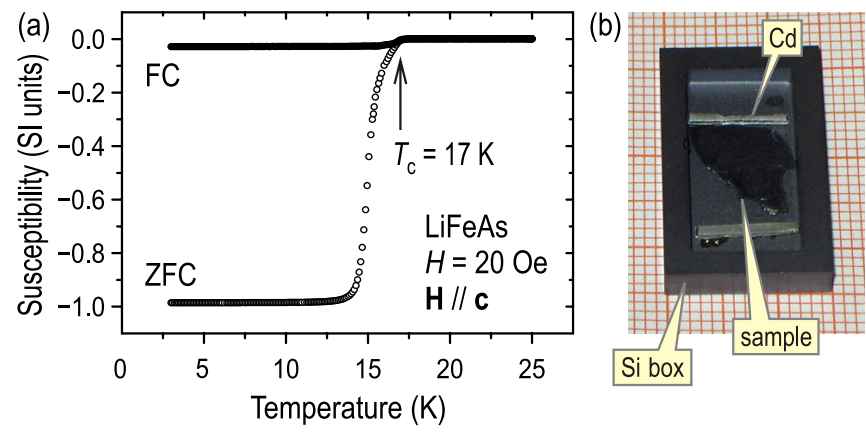

FIG. 1 (color online). (a) Magnetic susceptibility of LiFeAs, measured upon warming after cooling in magnetic field (FC) and in zero field (ZFC). (b) Photo of the sample prepared for SANS measurements inside the single-crystalline silicon box (see text). 
sample, was $\sim 17 \mathrm{~K}$, as shown in Fig. 1(a). In order to avoid any exposure of the sample to air during mounting into the cryostat, it was sealed inside a single-crystalline $\mathrm{Si}$ box [Fig. 1(b)] in Ar atmosphere. The box was essentially transparent to neutrons due to the low absorption and incoherent neutron-scattering cross sections of $\mathrm{Si}$. To mark the position of the sample inside the sealed box with respect to the neutron beam, two stripes of highly neutron-absorbent $\mathrm{Cd}$ were placed above and below it.

The SANS experiment was carried out using the D11 instrument at the Institut Laue-Langevin in Grenoble. The applied field was approximately parallel to the incident cold-neutron beam with a wavelength $\lambda=8 \AA$ and the full width at half maximum (FWHM) spread $\Delta \lambda / \lambda=10 \%$. The diffracted neutrons were collected by a $2 \mathrm{D}$ multidetector behind the sample. The vortex lattice (VL) was prepared in the sample by applying the desired field above $T_{c}$ and subsequent field cooling to $2 \mathrm{~K}$. Background measurements were carried out at $20 \mathrm{~K}\left(>T_{c}\right)$ and subtracted from the field-cooled foreground measurements.

Two representative diffraction patterns measured in magnetic fields $H=0.25$ and $0.5 \mathrm{~T}$ are shown in Figs. 2(a) and 2(b). A distinct ring of scattering is seen in both panels. The absence of distinct Bragg peaks indicates a lack of long-range orientational order of the VL. The $0.25 \mathrm{~T}$ image is a sum of 10 individual diffraction patterns measured at different rocking angles between $-8^{\circ}$ to $8^{\circ}$, whereas the $0.5 \mathrm{~T}$ image was measured at zero rocking angle only. At higher fields, the scattering intensity is decreased, but a peak can be seen in the angle-averaged data up to $H=1.5 \mathrm{~T}$, as shown in Fig. 2(c). For all fields, the fitted peak position agrees within the experimental error with the expected radius of the diffraction ring $q_{\Delta}=$ $4 / a_{\Delta} \sqrt{3}$ (shown by small vertical arrows), calculated for a perfect triangular VL with lattice parameter $a_{\Delta}=$ $\sqrt{2 \phi_{0} / H \sqrt{3}}$, where $\phi_{0}$ is the magnetic-flux quantum.

So far, these results are similar to those reported for electron-doped 122 compounds [9,12]. The first essential difference is illustrated in Fig. 2(d), which shows rocking curves with half width at half maximum (HWHM) of $3.2 \pm$ $0.5^{\circ}$, measured at $0.25 \mathrm{~T}$. They represent the angular dependence of the diffracted intensity on the left and right sides of the diffraction ring. Despite the clear intensity variation, vortex pinning causes the rocking curves to remain broad, and using the HWHM we estimate the longitudinal correlation length of the VL as $\zeta_{\|} \approx 0.8 \mu \mathrm{m}$.

Conversely, in previous SANS experiments on both underdoped [9] and overdoped [12] $\mathrm{BaFe}_{2-x} \mathrm{Co}_{x} \mathrm{As}_{2}$, the rocking curves were much broader and extended beyond the measurable range. Our observations therefore indicate an improvement in the longitudinal VL ordering and a decrease of the typical pinning forces in the absence of chemical dopants. But the fact that we did not observe any long-range orientational VL order even after oscillating the field value by $1 \%$ and $10 \%$ during in-field cooling suggests that the pinning in our sample is still not negligible.
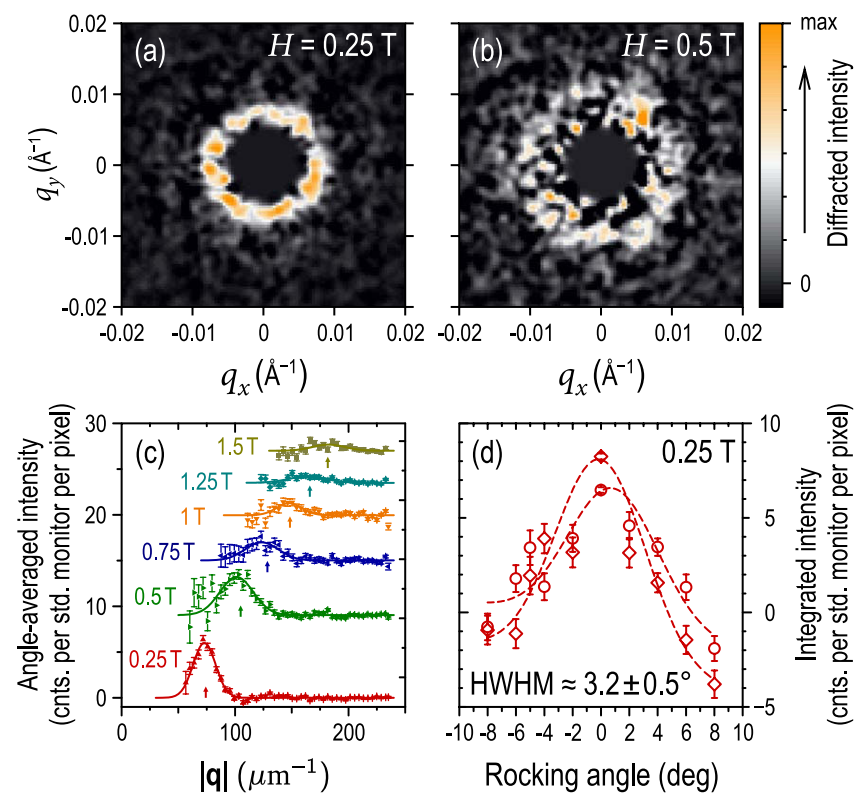

FIG. 2 (color online). (a),(b) SANS diffraction patterns measured at $H=0.25$ and $0.5 \mathrm{~T}$, respectively. The $0.25 \mathrm{~T}$ data are summed up over the rocking curve from $-8^{\circ}$ to $8^{\circ}$, whereas the $0.5 \mathrm{~T}$ data are shown for the zero rocking angle. Both data sets are smoothed with a 3-pixel FWHM Gaussian filter. (c) Angleaveraged diffracted intensity as a function of momentum transfer $|\mathbf{q}|$, measured at different magnetic fields between 0.25 and 1.5 T. For clarity, the zero line of each curve is offset from the one below it. Vertical arrows show the expected peak positions for a perfect triangular lattice $q_{\triangle}$. Solid lines are Gaussian fits. (d) Averaged intensities on the left $(\diamond)$ and right $(\bigcirc)$ sides of the ring as functions of the rocking angle, measured at $H=0.25 \mathrm{~T}$.

Now let us turn to the quantitative determination of some important SC properties of LiFeAs. The integrated intensity $I$ corresponding to $1 / 6$ of the diffraction ring (one Bragg spot of a triangular VL), obtained from the rocking curve, is proportional to the modulus squared of the VL form factor $F(q, T)$ [14], i.e., the Fourier transform of the 2D magnetic-flux modulation within the sample. As our measured intensity mainly originates from the first-order Bragg spots at a distance $q \approx q_{\Delta}$ from the origin, we limit our considerations to the first-order VL form factor,

$$
I=2 \pi V \Phi(\gamma / 4)^{2} \lambda^{2} \phi_{0}^{-2} q^{-1}|F(q, T)|^{2} .
$$

Here, $V$ is the sample volume, $\Phi$ is the neutron flux density, and $\gamma$ is the magnetic moment of the neutron in nuclear magnetons. By varying the magnetic field and, consequently, $q_{\Delta}$, we can thus study the $q$ dependence of the form factor, assuming that the rocking curve width is both field and temperature independent.

In the simplest Ginzburg-Landau model valid for superconductors with large $\kappa=\lambda / \xi \gg 1$, and in small magnetic fields $H \ll H_{c 2}$, the form factor can be expressed in terms of the temperature-dependent penetration depth $\lambda_{a b}(T)$ and the SC coherence length $\xi_{a b}(T)$ [15] (for brevity, the index $a b$ will not be used), 


$$
F(q, T)=\frac{H g K_{1}(g)}{1+[\lambda(T) q]^{2}}, \quad g=\sqrt{2} \frac{\xi(T)}{\lambda(T)} \sqrt{1+[\lambda(T) q]^{2}} .
$$

Here, $K_{1}(g)$ is the modified Bessel function of the second kind. Because the band structure of LiFeAs can potentially support two-gap SC [4], we resort to the following analytical two-gap model to describe the temperature dependence of the penetration depth,

$$
\frac{1}{\lambda^{2}(T)}=I_{1}\left[1-M\left(\frac{\Delta_{1}(T)}{k_{B} T}\right)\right]+I_{2}\left[1-M\left(\frac{\Delta_{2}(T)}{k_{B} T}\right)\right],
$$

where the function $M$ is an accurate approximation [16] for the temperature-dependent quasiparticle effects, and the constants $I_{1,2}$ depend only on the band structure and can be expressed as simple integrals over the FS $[16,17]$. The $T$ dependence of the SC gap is approximated by [18]

$$
\Delta(T)=\Delta_{0} \tanh \left(\frac{\pi}{2} \sqrt{T_{c} / T-1}\right) .
$$

Consequently, because in the weak-coupling limit the SC coherence length is inversely proportional to the energy gap [19], the temperature evolution of $\xi$ will be given by

$$
\xi(T)=\xi(0)\left[\tanh \left(\frac{\pi}{2} \sqrt{T_{c} / T-1}\right)\right]^{-1} \propto 1 / \Delta(T) .
$$

Substituting expressions (3)-(5) into Eq. (2), we obtain the final formula for fitting the SANS data.

Following the methodology of Ref. [20], we start with the magnetic field dependence of the low- $T$ form factor that is shown in Fig. 3(a). Because at $T=2 \mathrm{~K}$ the values of $\xi$ and $\lambda$ can be considered equal to their $T=0$ limits, Eq. (2) can be applied directly to the field-dependent data (solid line in the figure) to extract the values of $\lambda_{a b}(0)=$ $210 \pm 20 \mathrm{~nm}$ and $\xi_{a b}(0)=7 \pm 2 \mathrm{~nm}(\kappa=29 \pm 7)$. The obtained value of $\lambda_{a b}$ agrees with the results of a muonspin rotation $(\mu \mathrm{SR})$ measurement [21], which yielded $\lambda_{a b}(0)=195$ and $244 \mathrm{~nm}$ for two samples of $\mathrm{Li}_{1+\delta} \mathrm{FeAs}$ with $T_{c}=16$ and $12 \mathrm{~K}$, respectively. Our value of $\xi_{a b}(0)$, however, is likely to be overestimated with respect to that obtained from upper-critical-field measurements [22], which is $\xi_{a b}(0)=\sqrt{\phi_{0} / 2 \pi H_{c 2}^{\perp}} \approx 2-4 \mathrm{~nm}$. Such overestimation can result either from a finite-width $T_{c}$ distribution in our large sample or from the field-induced disorder of the VL expected in the Bragg glass model [23].

Next, we turn to the temperature evolution of the form factor. As follows from Eq. (2), for $\lambda(T) q \gg 1, F(q, T) \propto$ $\lambda^{-2}(T)$. The scattered intensity therefore scales $\propto \lambda^{-4}$. In Fig. 3(b), the measured integrated intensity is plotted versus temperature, and the vertical axis is scaled to the value of $1 / \lambda^{4}(0)$ that resulted from the low- $T$ fit of the form factor. By fixing $\lambda(0)$ and $\xi(0)$ to the values found previously, we can now fit the two SC gaps $\Delta_{1,2}(0)$ and the coefficients $I_{1,2}$, using $|F(q, T)|^{2}$ as the fitting function. It turns out that independently of the parameter initialization, the fit converges to a single value of the gap $\Delta_{1}=\Delta_{2}=$
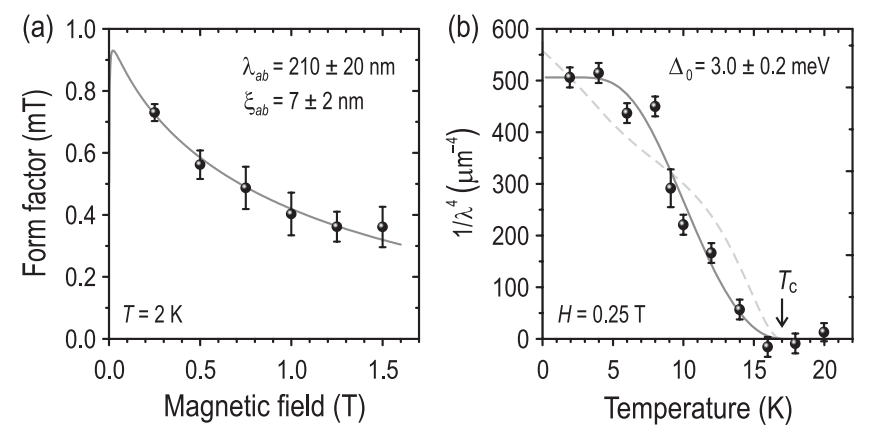

FIG. 3. (a) Magnetic field dependence of the VL form factor at $T=2 \mathrm{~K}$, fitted to Eq. (2). (b) Temperature dependence of $|F(q, T)|^{2}$ at $H=0.25 \mathrm{~T}$. The vertical axis is scaled to the value of $\lambda^{-4}(0)$ that resulted from the form-factor fit in (a). The dashed line shows $\lambda^{-4}(T)$ for the $d$-wave gap.

$3.0 \pm 0.2 \mathrm{meV}$. This value corresponds to the ratio $2 \Delta / k_{B} T_{c}=4.1 \pm 0.3$, approaching the weak-coupling limit of 3.53 predicted by the BCS theory of conventional superconductivity [24]. For comparison, $\lambda^{-4}(T)$ corresponding to a $d$-wave gap is also shown in the same figure, producing a poor fit. This essentially excludes the possibility of two-gap SC or gap nodes in LiFeAs.

Now we compare these results with those of ARPES, to establish their relationship to the microscopic electronic properties, such as band dispersion and the SC gap. An analysis of the leading edge shift along the FS contours implies an isotropic gap for every FS sheet [4]. To quantify the low- $T$ gap value $\Delta_{0}$, we employed the Dynes-function fitting procedure [25] to the ARPES spectra measured on the double-walled electronlike $M$ barrel [Figs. 4(a) and 4(b)]. The energy distribution curves integrated in a wide momentum window along the FS radius (IEDCs), measured in the SC state below $1 \mathrm{~K}$ and in the normal state at $23 \mathrm{~K}$, are shown in Fig. 4(c). In order to reveal the true shape of the spectrum in the SC state, the low- $T$ IEDC was normalized by the Fermi-function-corrected normal-state spectrum, as shown in Fig. 4(d). The good quality of the Dynes-function fit confirms the robustness of such normalization. The resulting low-temperature value of $\Delta_{0}^{\text {ARPES }}=$ $3.1 \pm 0.3 \mathrm{meV}$ is in perfect agreement with that extracted above from the temperature dependence of $\lambda_{a b}$.

The knowledge of the band dispersion together with the SC gap allows the calculation of macroscopic properties in the SC state with no adjustable parameters. The superfluid density at $T \rightarrow 0$ is proportional to the integral of Fermi velocity $v_{F}$ along the FS perimeter $[16,17,26]$, and in the clean limit,

$$
\frac{1}{\lambda_{a b}^{2}}=\frac{e^{2}}{2 \pi \varepsilon_{0} c^{2} h L_{c}} \oint_{\mathrm{FS}} v_{F} d k,
$$

where $\varepsilon_{0}, h, e, c$ are physical constants and $L_{c}$ is the $c$-axis lattice parameter. Although the FS of LiFeAs consists of several electronlike and holelike sheets [4], for the evaluation of the integral (6) the renormalized Fermi velocity, extracted from ARPES data, can be well approximated by 

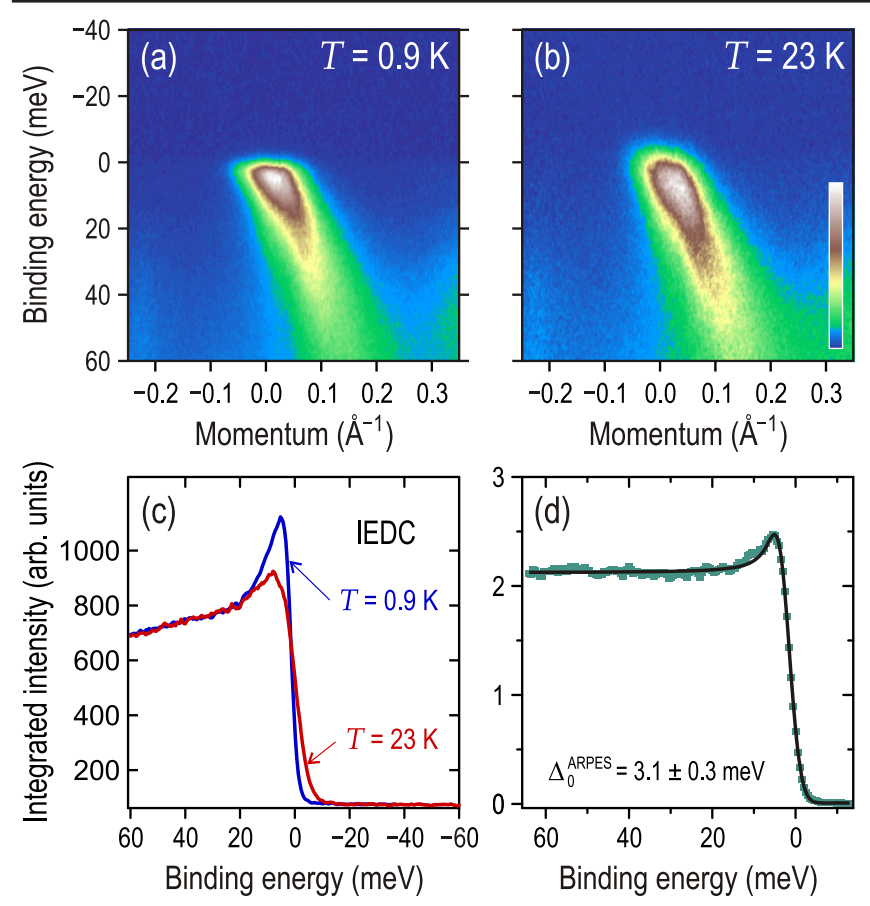

FIG. 4 (color online). ARPES spectra of LiFeAs measured on the double-walled electronlike $M$ barrel in the SC (a) and normal (b) states. (c) The integrated energy distribution curves (IEDCs) of the same spectra. (d) The low-temperature IEDC after normalization, fitted to the Dynes function.

its average value of $\hbar\left\langle v_{F}\right\rangle=0.3 \pm 0.03 \mathrm{eV} \AA$. For the experimental $\mathrm{LiFeAs}$ band structure, this formula yields $\lambda_{a b}^{\mathrm{ARPES}}=172 \pm 20 \mathrm{~nm}$, which is only slightly lower than our directly measured value. Similarly, at $T \rightarrow 0$, the BCS coherence length is proportional to the ratio of Fermi velocity to gap magnitude, $\xi_{a b}^{\mathrm{ARES}}(0)=\hbar\left\langle v_{F}\right\rangle / \pi \Delta_{0}$ [24], which equals $3.2 \pm 0.4 \mathrm{~nm}$ in our case. This corresponds to the upper critical field $H_{c 2}^{\perp}=32 \pm 8 \mathrm{~T}$, in agreement with direct measurements [22].

In summary, we have evaluated several important SC parameters of LiFeAs from two complementary experiments. We have demonstrated that its order parameter is isotropic, and in contrast to the higher- $T_{c}$ ferropnictides [16] is characterized by a single SC gap $\Delta_{0}=3.0 \pm$ $0.2 \mathrm{meV}$. This value is close to the BCS limit of $1.76 k_{B} T_{c}$, which indicates that LiFeAs is a weakly coupled single-gap superconductor, similar to conventional metals.

We thank B. Büchner and B. Keimer for their helpful suggestions and support, and acknowledge discussions with L. Boeri and S. A. Kuzmichev. Sample growth was supported by the DFG project BE 1749/12. SANS experiments were done with financial assistance from the EPSRC UK and MaNEP. I. V. M. acknowledges support from the Ministry of Science and Education of Russian Federation under the State contract P-279. ARPES spectra were measured with the " 13 -ARPES" end station, using radiation from the BESSY II synchrotron in Berlin.

Note added in proof.-Additional low- $T$ contributions to some thermodynamic quantities have been recently asso- ciated with a second, much smaller, SC gap [27]. Our data exclude such a gap both on the $M$ - and large $\Gamma$-centered barrels; hence, this contribution can only originate from the inner $\Gamma$ bands with a van Hove singularity near the Fermi level [4], which have a negligible effect on the superfluid density because of the small $v_{F}$.

*Corresponding author. d.inosov@fkf.mpg.de

[1] Y. Kamihara et al., J. Am. Chem. Soc. 130, 3296 (2008); M. Rotter et al., Phys. Rev. Lett. 101, 107006 (2008).

[2] X. C. Wang et al., Solid State Commun. 148, 538 (2008); M. J. Pitcher et al., Chem. Commun. (Cambridge) 45 (2008) 5918; J. H. Tapp et al., Phys. Rev. B 78, 060505(R) (2008).

[3] I. A. Nekrasov, Z. V. Pchelkina, and M. V. Sadovskii, JETP Lett. 88, 543 (2008); L. Zhang et al., Phys. Rev. B 78, 174520 (2008); D. J. Singh, ibid. 78, 094511 (2008).

[4] S. V. Borisenko et al., arXiv:1001.1147.

[5] P. Jeglič et al., arXiv:0912.0692 [Phys. Rev. B (to be published)].

[6] R. A. Jishi and H. M. Alyahyaei, Adv. Condens. Matter Phys. (2010) 804343.

[7] I. Mazin et al., Phys. Rev. Lett. 101, 057003 (2008); K. Kuroki et al., ibid. 101, 087004 (2008); D. S. Inosov et al., Nature Phys. 6, 178 (2010).

[8] A. A. Kordyuk et al., arXiv:1002.3149.

[9] M. R. Eskildsen et al., Phys. Rev. B 79, 100501(R) (2009); L. Ya. Vinnikov et al., JETP Lett. 90, 299 (2009).

[10] Y. Yin et al., Phys. Rev. Lett. 102, 097002 (2009).

[11] M. A. Tanatar et al., Phys. Rev. B 79, 180508(R) (2009); R. Prozorov et al., ibid. 80, 174517 (2009).

[12] D. S. Inosov et al., Phys. Rev. B 81, 014513 (2010).

[13] J. T. Park et al., Phys. Rev. Lett. 102, 117006 (2009); D. S. Inosov et al., Phys. Rev. B 79, 224503 (2009).

[14] D. K. Christen et al., Phys. Rev. B 15, 4506 (1977).

[15] J.R. Clem, J. Low Temp. Phys. 18, 427 (1975); A. Yaouanc, P.D. de Réotier, and E. H. Brandt, Phys. Rev. B 55, 11107 (1997).

[16] D. V. Evtushinsky et al., New J. Phys. 11, 055069 (2009).

[17] R. Khasanov et al., Phys. Rev. Lett. 102, 187005 (2009).

[18] F. Gross et al., Z. Phys. B 64, 175 (1986).

[19] L. Benfatto, A. Toschi, S. Caprara, and C. Castellani, Phys. Rev. B 66, 054515 (2002).

[20] J. S. White et al., J. Phys. Condens. Matter 20, 104237 (2008).

[21] F. L. Pratt et al., Phys. Rev. B 79, 052508 (2009).

[22] Y. J. Song et al., arXiv:1002.2249.

[23] T. Klein et al., Nature (London) 413, 404 (2001).

[24] J. Bardeen, L. N. Cooper, and J. R. Schrieffer, Phys. Rev. 108, 1175 (1957).

[25] R. C. Dynes, V. Narayanamurti, and J. P. Garno, Phys. Rev. Lett. 41, 1509 (1978); D. V. Evtushinsky et al., Phys. Rev. B 79, 054517 (2009).

[26] B. S. Chandrasekhar and D. Einzel, Ann. Phys. (N.Y.) 505, 535 (1993).

[27] F. Wei et al., arXiv:1004.0854 [Phys. Rev. B (to be published)]; K. Sasmal et al., Phys. Rev. B 81, 144512 (2010). 